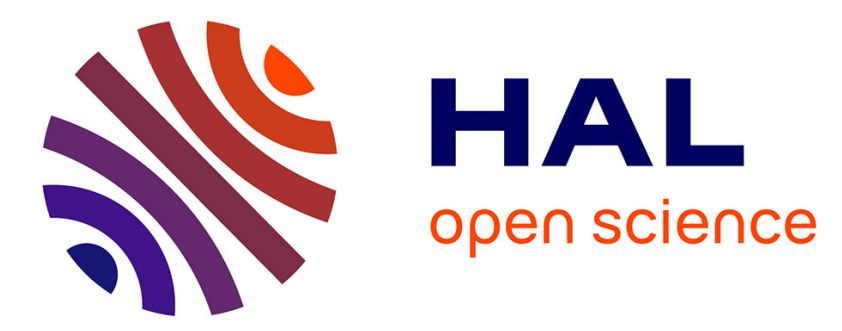

\title{
5-axis local corner rounding of linear tool path discontinuities
}

Xavier Beudaert, Sylvain Lavernhe, Christophe Tournier

\section{To cite this version:}

Xavier Beudaert, Sylvain Lavernhe, Christophe Tournier. 5-axis local corner rounding of linear tool path discontinuities. International Journal of Machine Tools \& Manufacture, 2013, 73, pp.9 - 16. 10.1016/j.ijmachtools.2013.05.008 . hal-00843635

\section{HAL Id: hal-00843635 \\ https://hal.science/hal-00843635}

Submitted on 11 Jul 2013

HAL is a multi-disciplinary open access archive for the deposit and dissemination of scientific research documents, whether they are published or not. The documents may come from teaching and research institutions in France or abroad, or from public or private research centers.
L'archive ouverte pluridisciplinaire HAL, est destinée au dépôt et à la diffusion de documents scientifiques de niveau recherche, publiés ou non, émanant des établissements d'enseignement et de recherche français ou étrangers, des laboratoires publics ou privés. 


\title{
5-axis local corner rounding of linear tool path discontinuities
}

\author{
Xavier Beudaert, Sylvain Lavernhe, Christophe Tournier* \\ LURPA, ENS Cachan, Université Paris Sud \\ 61 av du Président Wilson, 94235 Cachan, France \\ name@lurpa.ens-cachan.fr
}

\begin{abstract}
5-axis high speed machine tools are widely used in industry. Most of the time, the tool path is described with linear segments (G1) which leads to tangency discontinuities between blocks. The aim of this paper is to smooth the tool path geometry using a 5-axis corner rounding method suitable for acceleration and jerk limited feedrate interpolation.

Several methods have been developed in 3-axis but 5-axis corner rounding is still a challenge due to the difficulties linked to the smoothing of the orientation. The proposed corner rounding model allows to control precisely the contour and orientation tolerances in the workpiece coordinate system for 3 and 5-axis tool path. To smooth the tool tip position and the tool orientation in the corner, 5 -axis tool paths are represented by two B-Spline curves.

The main difficulty is the connection between the initial tool path and the newly inserted smoothing portion. To obtain a smooth connection of the orientation a parametrization spline is required to link the bottom and top B-Spline parameters. This algorithm is integrated to a feedrate interpolator which controls a 5-axis milling machine equipped with an Open CNC.
\end{abstract}

Keywords: 5-axis, G1 discontinuity, smoothing, feedrate, jerk, CNC, corner rounding

\section{Introduction}

Whilst major improvements in Computer Aided Manufacturing (CAM) and machine tools design have been observed, advances in the design of Computer Numerical Control (CNC) have been limited. Even if polynomial and B-Spline representations of the tool paths have been introduced in the industrial CNCs, most of the milling tool paths are still defined by G1 blocks (linear interpolation). However, this description generates tangential discontinuities at each transition between the linear segments. Considering that the machine tool axes have acceleration and jerk limitations, the only solution to avoid a full stop at each transition is to smooth the geometry to obtain a continuous tool path. That means that the $\mathrm{CNC}$ has to modify the geometry given in the part program using user tolerance parameters.

The feedrate interpolation is closely linked to the geometry of the tool path. It is possible to use a decoupled approach [1] which will first design a continuous tool path geometry and then find an admissible motion law along this fixed geometry. The other solution is to treat the problem globally with a combined approach which will compute both the geometry and the motion law at once. This approach requires some hypotheses about the feedrate and acceleration which are not always verified. The rest of the paper will focus on the decoupled approach. Once the geometry is smoothed, the feedrate planning can be performed using, for example, the previously developed Velocity Profile Optimization [2].

\footnotetext{
${ }^{*}$ Corresponding author
}

Depending on the context, two main methods are available to smooth the tool path geometry, respectively the global smoothing and the local smoothing. If a portion of the tool path is composed of a high density of short segments, it is possible to approximate all the segments by a curve. On the other hand, if the tool path is composed of long segments, it is important to follow precisely these segments and thus each transition has to be locally smoothed. This paper focuses on the latter problem which has been studied by several authors for 3-axis tool paths but which is still a challenge in 5-axis milling.

Global smoothing has been studied in 3-axis where the main difficulty is to respect a given contour tolerance around the programmed segments, see Siemens compressor [3] and other publications like [4-6].

In 5-axis, global smoothing techniques are also widely studied. Different solutions can be used to smooth the orientation: quaternions $[7,8]$, spherical B-Splines [9, 10], position and orientation curves [11, 12], drive movement smoothing [13-15].

Global smoothing techniques cannot be applied for local corner rounding because several requirements cannot be fulfilled. First the contour and orientation modifications have to be controlled locally and precisely avoiding oscillations. Second, the corner rounding curve has to be connected to the initial tool path. Thus our research not only brings new contribution for 5 -axis local tolerance management but also for the orientation connection of two portions of 5 -axis tool path defined by BSpline curves.

Local smoothing has been widely studied in 3-axis. Indeed, different methods which give similar results are detailed in the literature. They all give a satisfying corner rounding curve in

December 13, 2012 
terms of contour error and continuity management. Indeed $G^{2}$ continuity is assured to allow jerk limited feedrate interpolation. Yutkowitz and Chester [16] registered a Siemens patent describing a 3-axis corner rounding method based on two $4^{\text {th }}$ order polynomial curves. Erkorkmaz et al. [17] use a $5^{\text {th }}$ order polynomial curve to round the corner. A cubic B-Spline with 8 control points is used by Pateloup et al. [18] whereas a cubic BSpline with 5 control points is used by Zhao et al. [19]. Ernesto and Farouki [20] employed a Bezier conic and optimized the feedrate along the curve under acceleration bounds. Bi et al. [21] used two cubic Bezier curves to round the corner. None of these papers tried to extend their solution to 5-axis tool path which brings new challenges. To the best of our knowledge, no previously published work has tackled the problem of 5-axis corner rounding.

It is possible to broaden the context of corner rounding. Indeed, most of the publications are focused on the need for the $\mathrm{CNC}$ to round corner using generally a small tolerance of the order of a few hundredths of a millimetre. However, as in Pateloup et al. [18], it can also be applied in a CAM context with a fairly large geometrical deviation. Within the framework of the Step-NC standard development which is a high level intelligent programming environment, it could be useful to be able to locally round a CAM tool path. The solution presented in this paper is multi-scale and can be applied to both contexts.

The aim of this paper is to propose a new 5-axis corner rounding method. 5-axis corners are rounded using two B-Spline curves which define the tool tip and tool orientation. This solution is especially suited for flank milling as the contour and orientation tolerances are precisely controlled. The main difficulty is coming from the orientation smoothing and more specifically from the connection between the initial tool path and the newly inserted smoothing portion. The orientation connection problem between two 5-axis portions is addressed in details and solved using a third B-Spline curve for the parametrization of the tool orientation.

The rest of the paper is organized as follows. First, the 5axis corner rounding geometry is presented in section 2 . Then, the problem of the continuous variation of the orientation is addressed in section 3. Experiments and simulations are carried out in section 4 to demonstrate the efficiency of the proposed method. Finally, the paper is concluded in section 5 .

\section{5-axis corner rounding method}

After an analysis of the different orientation smoothing solutions, a representation of the 5-axis tool path based on two curves is chosen. The typical 3-axis corner rounding problem has to be solved using a model which is then extended for the 5 -axis corner rounding problem. Finally, it is shown that the main difficulty comes from the orientation connection which is an original problem.

\subsection{Analysis of the different orientation smoothing solutions}

Several solutions are available to smooth the tool orientation. The solutions based on a modification of the tool path in the Machine Coordinate System [13-15] have two main drawbacks. First, it is difficult to control the geometrical deviation on the workpiece resulting from a modification of an axis movement. Then, those solutions require the machine kinematical transformation, so they are dedicated to a specific machine tool. That is why solutions based on a modification of the tool path in the Workpiece Coordinate System are preferred here.

Rotation smoothing can be carried out using spherical BSplines on the unit sphere $[9,10]$ or smoothing the orientation components $i j k$. Other solutions using quaternion have also been developed [7]. But here again, the use of these techniques does not allow a precise control of the resulting workpiece geometry. Another important need for corner rounding is to avoid the oscillations which could be generated by these methods.

To avoid the oscillations and to control precisely the geometrical deviations, a representation of the 5-axis tool path by two curves is chosen. The bottom curve defines the locus of the tool tip locations and the top curve defines the locus of a second point belonging to the tool axis [11]. Thus the corner rounding is performed on both $3 \mathrm{D}$ curves which leads to apply a 3-axis corner smoothing method twice.

\subsection{3-axis corner rounding method}

As we have just seen, 5-axis corners are defined by two curves which have to be rounded as for 3-axis corner rounding problems. The aim of the 3 -axis corner rounding algorithm is to obtain a $G^{2}$ continuous geometry along which the jerk limited feedrate profile will further be computed, Fig. 1.

The modified geometry has to satisfy the specified contour tolerance $\varepsilon$. Many 3-axis methods have been proposed in the literature, the model presented here is simple and suitable for the extension to 5-axis.

By construction with 3 control points aligned, the connection between the rounding curve and the previous and following segments is $G^{2}$. The definition of the cubic B-Spline used to round the corner is given in Eq. 1 with $\mathbf{P}_{i}$ the control points, $B_{i 3}$ the basis functions and $\mathbf{u}=\left[\begin{array}{lllllllll}0 & 0 & 0 & 0 & 0.5 & 1 & 1 & 1 & 1\end{array}\right]$ the knot sequence, see Fig. 1.

$$
\mathbf{C}(u)=\sum_{i=0}^{4} B_{i 3}(u) \mathbf{P}_{i}
$$

Taking Eq. 1 and the definition of the basis functions $B_{i 3}$, Eq. 2 can be obtained (further details are given in Appendix A). With this construction of a cubic B-Spline with 5 control points, the point $\mathbf{C}(u=0.5)$ does not depend on the control points $\mathbf{P}_{0}$ and $\mathbf{P}_{4}$. The points $\mathbf{P}_{1}$ and $\mathbf{P}_{3}$ are positioned symmetrically so the distance $L_{1}=\mathbf{P}_{1} \mathbf{P}_{2}=\mathbf{P}_{2} \mathbf{P}_{3}$ (Fig. 1). This curve definition allows to have the maximum contour error exactly in the middle of the rounding curve at $u=0.5$.

$$
\mathbf{C}(u=0.5)=\frac{1}{4} \mathbf{P}_{1}+\frac{1}{2} \mathbf{P}_{2}+\frac{1}{4} \mathbf{P}_{3}
$$

Finally, an advantage of this model is to have a simple equation of the contour error as a function of the corner angle $\alpha$ and the length $L_{1}$, Eq. 3 . 


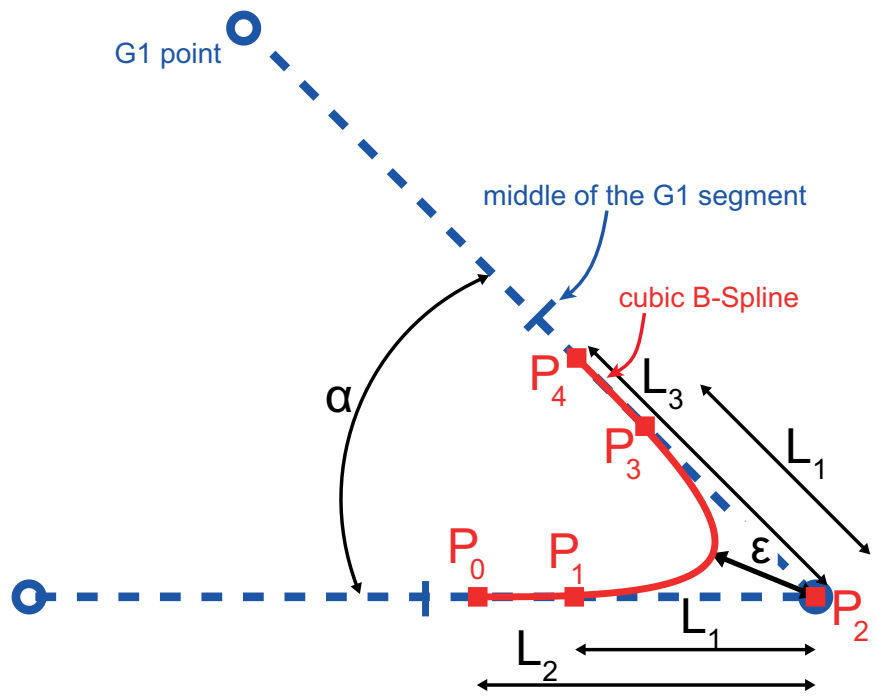

Figure 1: 3-axis corner rounding method

$$
\varepsilon=\frac{1}{2} L_{1} \cos \left(\frac{\alpha}{2}\right)
$$

The contour error constraint allows to determine the length $L_{1}$, but the lengths $L_{2}$ and $L_{3}$ can still be optimized. It will be shown later that when this model is used on the top curve, the lengths $L_{2}$ and $L_{3}$ are defined by the interpolation of orientation on the segments.

However, in 3-axis, $L_{2}=L_{3}$ is chosen to have a symmetrical corner. Thus the ratio between $L_{1}$ and $L_{2}$ is the last parameter to be optimized. To solve a similar problem, Pateloup et al. [18] used a criterion based on the curvature. Nevertheless, the best criterion for the optimization would be to minimize the machining time and thus it requires the use of a feedrate interpolation algorithm. A detailed presentation of this optimization is out of the topic of this paper. But numerical experiments have shown that $1.4 \leq \frac{L 2}{L 1} \leq 1.75$ gives satisfying results in terms of machining time minimization. Indeed, if $\frac{L 2}{L 1}$ is too close to 1 the connection between the curve and the segment is not smoothed enough. If $\frac{L 2}{L 1}=1, P_{0}=P_{1}$ and $P_{3}=P_{4}$ and a jerk limited feedrate interpolation would lead to zero feedrate because the connection would be $G^{1}$ and not $G^{2}$. Increasing the ratio more than 1.75 is useless regarding the machining time reduction. Another problem appears with small G1 segments. If the segments are too small, it is impossible to use the whole contour error tolerance. So the ratio is set to 1.4 and $L_{2}$ is chosen to use only half of the shortest segment in order to have independent corner rounding curves. Bi et al. [21] showed that it is possible to avoid this restrictive approach but if many short segments are used, a global interpolation method is probably more appropriate.

Finally, as it has been said in the introduction, this 3-axis model is multi-scale. Thus it can be useful both for small corner rounding carried out in the $\mathrm{CNC}$ or for larger corner rounding performed in the CAM stage.

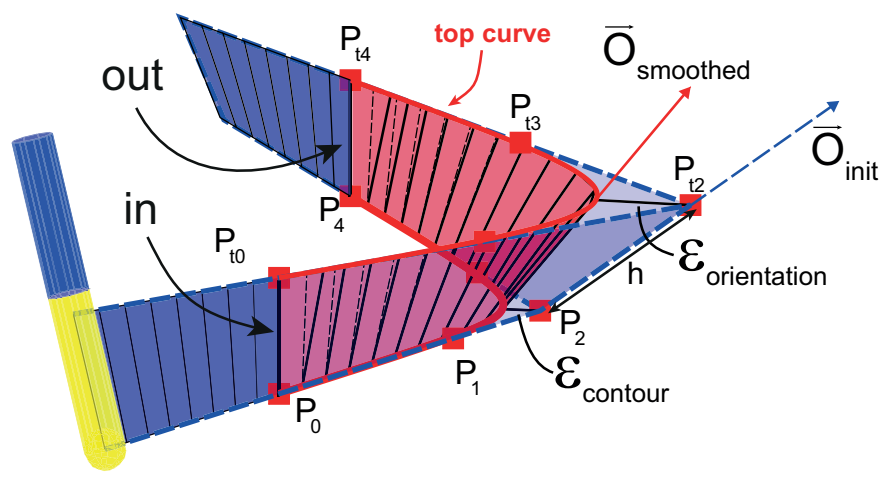

Figure 2: 5-axis corner rounding method

\subsection{5-axis corner rounding method}

Similarly to the tangency discontinuity problems encountered in 3-axis, 5-axis linear programming (G1) brings discontinuity problems on the position and orientation of the tool path. To the best of our knowledge, no extension to 5-axis corner rounding has been presented before.

The proposed model is based on the representation of the 5axis tool path by a bottom curve defining the tool tip movement and by a top curve defining the tool orientation. So the 3 -axis corner rounding model presented in previous section is applied to both 3D curves, see Fig. 2. The entrance (in) and exit (out) of the corner rounding portion are defined by the bottom curve. Thus the points $P_{t 0}$ and $P_{t 4}$ are positioned to respect the orientation interpolation on the linear segment. Then, the positions of $P_{t 1}$ and $P_{t 3}$ are given by the tolerance on the top curve.

This orientation tolerance $\varepsilon_{\text {orientation }}$ is well suited for tolerance management in flank milling. Indeed, it allows to specify the geometrical deviation in millimetres at the top of the workpiece with the given flank height $h$. The geometry of the smooth tool path is completely controlled in the Workpiece Coordinate System and the construction ensure the avoidance of oscillations.

A smooth surface containing the tool axis is thus defined by those two curves. Both curves are connected with $G^{2}$ continuity but a degree of freedom still exists for the tool orientation. As shown in Fig. 2 with the black dash lines, even if the surface connection is $G^{2}$, it is possible to have an important variation of the tool orientation at the entrance and exit of the rounding portion. The interpolation of the orientation in the rounding portion plays a key role for the smoothness of the tool path. This main difficulty for 5-axis corner rounding is studied in details in section 3 .

\subsection{Interpolation of the orientation}

Along the linear segments, the tool path is interpolated so that the orientation vector runs in a plane created by the start and end vectors. The rotation angle $\theta$ between the start vector $\mathbf{V}$ and an intermediate orientation respects the ratio between the angle and the linear displacement covered. The intermediate orientation vector $\mathbf{V}_{\text {rot }}$ is given by the Rodrigues' rotation formula, Eq. 4, with $\mathbf{k}$ the unit vector defining the axis of rotation (cross product of the start and end vectors). 


$$
\mathbf{V}_{r o t}=\mathbf{V} \cos \theta+(\mathbf{k} \times \mathbf{V}) \sin \theta+\mathbf{k}(\mathbf{k} \cdot \mathbf{V})(1-\cos \theta)
$$

In the rounding portion, the orientation of the tool is given by the difference of the top and bottom points, Eq. 5. So the orientation is not only given by the geometry of the curves which are fixed but also by the parametrization of the curves. Depending on the parametrization, the orientation is changing inside of the rounding portion and it can bring a severe orientation discontinuity at the connections with the linear segments.

$$
\operatorname{Ori}\left(u_{\text {bottom }}\right)=\frac{\mathbf{C}_{\text {top }}\left(u_{\text {top }}\right)-\mathbf{C}_{\text {bottom }}\left(u_{\text {bottom }}\right)}{\left\|\mathbf{C}_{\text {top }}\left(u_{\text {top }}\right)-\mathbf{C}_{\text {bottom }}\left(u_{\text {bottom }}\right)\right\|}
$$

\subsection{Parametrization spline}

The link between parameters $u_{t o p}$ and $u_{\text {bottom }}$ defines the tool orientation. For the rest of the paper, the bottom curve is parametrized according to its arc length. This parametrization is fixed, and the aim is to find the corresponding $u_{t o p}$ which will allow a smooth connection at the entry and exit of the rounding portion. Fig. 3 gives a schematic representation of the connection problem. The interpolation of the orientation on the previous segment is given by Eq. 4 so the derivatives at the end of the previous segment are fixed. To have a smooth transition, it is necessary to control the derivatives at the beginning of the rounding portion which are given by the link between $u_{t o p}$ and $u_{\text {bottom }}$. The easiest link is a linear parametrization $u_{\text {top }}=u_{\text {bottom }}$ but this parametrization leads to a zero feedrate at the connection as it will be demonstrated in the application. So an optimized cubic B-Spline parametrization curve is used to control the boundary conditions at the entry and exit of the rounding portion.

As the feedrate interpolation performed along this geometry considers the jerk limitations, it is necessary to control the connection up to the third derivatives. The equations are given and explained in Appendix B.

The important derivative is not the derivative with respect to the parameter of the curve $u$ but it is the derivative with respect to the path displacement $s$ (cumulative arc length of the tool tip movement). Indeed, this derivative called geometrical derivative is directly linked to the feedrate as it is shown in section 3.1.

\subsection{Generalization to the orientation connection problem}

The problem of orientation connection presented for the 5 -axis corner rounding can be generalized. Indeed, the same problem is encountered to connect two general 5-axis B-Spline tool paths, Fig. 4. For 5-axis machining, it is possible to use a polynomial tool path format $[3,11]$ but no attention was paid to the parametrization link between the bottom and top curves. Hence the default linear link $u_{t o p}=u_{\text {bottom }}$ generates a discontinuous variation of the tool orientation and thus a really low feedrate at the connection even if the curves have a good geometrical connection. Using a parametrization spline, section 3 presents a new contribution to obtain a smooth orientation connection.

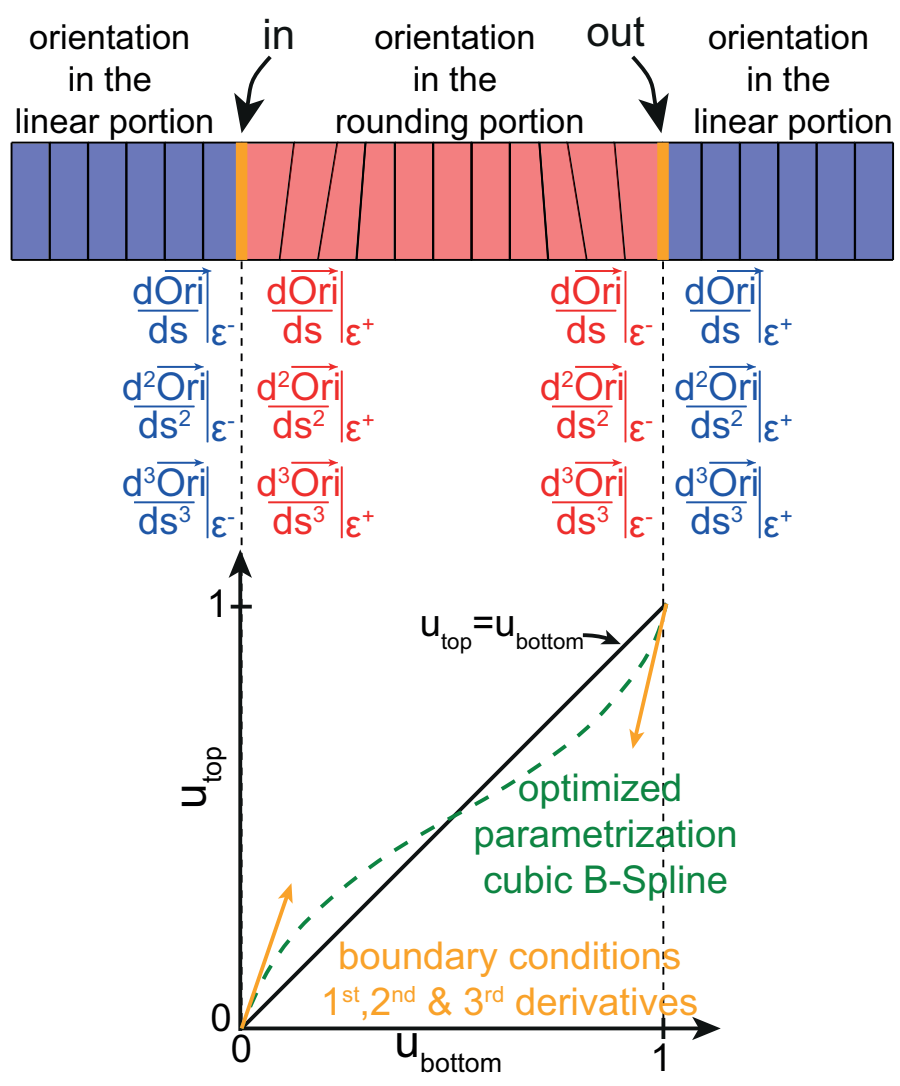

Figure 3: Parametrization spline for the orientation connection

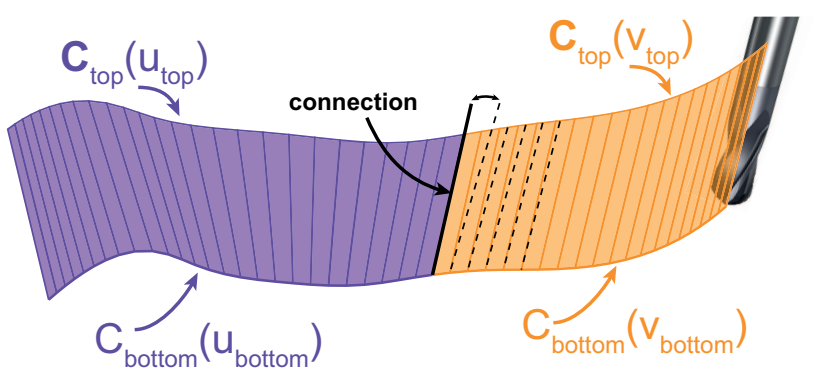

Figure 4: General orientation connection problem 


\section{Smooth orientation connection}

In the previous section, the 5-axis corner rounding model has been presented. It has been shown that it leads to a general problem of smooth orientation connection between two 5-axis tool path portions, see Fig. 4. Indeed, if the evolution of tool orientation at the connection is not smoothed enough, the jerk limited feedrate interpolation generates an important reduction of the feedrate. So first, it is important to understand why the feedrate is reduced and then a criterion is constructed to find an optimized orientation connection avoiding the feedrate slowdown.

\subsection{Approximation of the upper limit of the feedrate}

The feedrate is limited by the kinematical characteristics of the machine drives. The velocity, acceleration and jerk of the 5 drives $(\dot{\mathbf{q}}, \ddot{\mathbf{q}}, \dddot{\mathbf{q}})$ can be expressed as a function of the axis movement geometrical derivatives $\left(\mathbf{q}_{s}, \mathbf{q}_{s s}, \mathbf{q}_{s s s}\right)$ and of the motion law $(\dot{s}, \ddot{s}, \dddot{s})$, see Eq. 6-8. It is interesting to point out that these equations are valid for linear and rotary axes, which allows to compare the constraints on the 5 axes. $q=\left[\begin{array}{llll}X & Y Z & A & C\end{array}\right]$ is a vector containing the evolution of the geometry on the 5 axes in the Machine Coordinate System. $s$ is the path displacement, so $\dot{s}$ is the feedrate.

$$
\begin{gathered}
\dot{\mathbf{q}}=\frac{d \mathbf{q}}{d t}=\frac{d \mathbf{q}}{d s} \frac{d s}{d t}=\mathbf{q}_{s} \dot{s} \\
\ddot{\mathbf{q}}=\mathbf{q}_{s s} \dot{s}^{2}+\mathbf{q}_{s} \ddot{s} \\
\dddot{\mathbf{q}}=\mathbf{q}_{s s s} \dot{s}^{3}+3 \mathbf{q}_{s s} \dot{s} \ddot{s}+\mathbf{q}_{s} \dddot{s}
\end{gathered}
$$

Each drive has its own kinematical limits in terms of velocity, acceleration and jerk, respectively $\mathbf{V}_{\max }^{\text {axis }}, \mathbf{A}_{\max }^{\text {axis }}$ and $\mathbf{J}_{\max }^{\text {axis }}$. The role of the feedrate interpolator is to create the motion law respecting all the kinematical constraints (Eq. 9) along the tool path and especially the axis jerk [2]. The axis jerk often limits the actual feedrate, it is an important parameter to avoid excessive vibrations in high speed machining.

$$
|\dot{\mathbf{q}}| \leq \mathbf{V}_{\text {max }}^{\text {axis }} ;|\ddot{\mathbf{q}}| \leq \mathbf{A}_{\text {max }}^{\text {axis }} ;|\dddot{\mathbf{q}}| \leq \mathbf{J}_{\max }^{\text {axis }}
$$

The feedrate interpolation is a complex process but with some approximations it is possible to obtain an upper limit of the feedrate according to the velocity, acceleration and jerk constraints of the machine drives. This approximate feedrate limit is correct in the areas where the feedrate has to be decreased which are the most interesting areas for us. Indeed, during a feedrate slowdown, the feedrate is decreasing to a minimum and then increasing. So when the minimum feedrate is reached, the tangential acceleration $\ddot{s}$ and jerk $\dddot{s}$ are equal to zero which justifies the approximations.

Finally, the feedrate $\dot{s}$ has to be lower than the minimum of the programmed feedrate $F_{p r}$ and of the limitations given by the kinematical constraints of each axis of the machine tool, Eq. 10.

$$
\dot{s} \leq \min _{i=1 . .5}\left(F_{p r}, \frac{V_{\max }^{a x i s_{i}}}{\left|q_{s}\right|}, \sqrt{\frac{A_{\max }^{a x i s_{i}}}{\left|q_{s s}\right|}}, \sqrt[3]{\frac{J_{\max }^{\text {axis }}}{\left|q_{s s s}\right|}}\right)
$$

This upper limit of the feedrate has proven its efficiency in predicting the feedrate slowdowns, further information about its usefulness can be found in [13]. For a given machine, the parameters $\mathbf{V}_{\max }^{\text {axis }}, \mathbf{A}_{\text {max }}^{\text {axis }}$ and $\mathbf{J}_{\max }^{\text {axis }}$ are fixed so to increase the feedrate, it is necessary to minimize the magnitude of the axis movement geometrical derivatives. But all this is based on the axis movement in the Machine Coordinate System which depends on the geometry of the machine, workpiece setup, tool length... To obtain a solution independent of these parameters, a criterion based only on the Workpiece Coordinate System has to be constructed.

\subsection{Criterion based on the norm of the third geometrical derivatives of the tool orientation}

The feedrate is limited by the machine drive kinematical limits in velocity, acceleration and jerk. However, the most limiting parameter is the axis jerk. So according to Eq. 10, the main objective would be to minimize the third geometrical derivatives $\mathbf{q}_{\text {sss }}$. As the connection problem comes from the orientation, the tool tip is fixed and the tool orientation will be optimized.

The link between the Machine Coordinate System and the Workpiece Coordinate System is given by the kinematical transformation which is non-linear but which preserves the continuity (trigonometric functions). Thus in the Workpiece Coordinate System the last degree of freedom is the evolution of the tool orientation on the surface containing the locus of tool axis. In the Workpiece Coordinate System, the orientation of the tool is represented by the orientation components $i j k$. Finally, the minimization of $\mathbf{q}_{s s s}$ is linked to the minimization of the third geometrical derivatives of the tool orientation. As no component should be privileged, a criterion based on the norm of the third derivatives of the tool orientation with respect to the path displacement is constructed, Eq. 11 .

$$
\text { Criterion }=\sqrt{\left(\frac{d^{3} i}{d s^{3}}\right)^{2}+\left(\frac{d^{3} j}{d s^{3}}\right)^{2}+\left(\frac{d^{3} k}{d s^{3}}\right)^{2}}
$$

\subsection{Optimized orientation connection}

Using the criterion previously defined, it is possible to run the optimization of the orientation connection in the Workpiece Coordinate System. The objective is to minimize the criterion in order to have the smoothest connection and thus the highest feedrate. As it is explained in section 2.5, the last degree of freedom is the parametrization of the top curve. As it has been shown in Fig. 3, a parametrization spline is used to link the bottom and top parameters. To compute the discrete third derivative, a backward Euler method is used so the third derivative at one point is based on the three previous points. For the 5 -axis corner rounding problem, to control the boundary conditions the three first and last points of the parametrization spline are optimized using a Newton-Raphson method. The procedure starts by finding the best first point, then this point is fixed and 


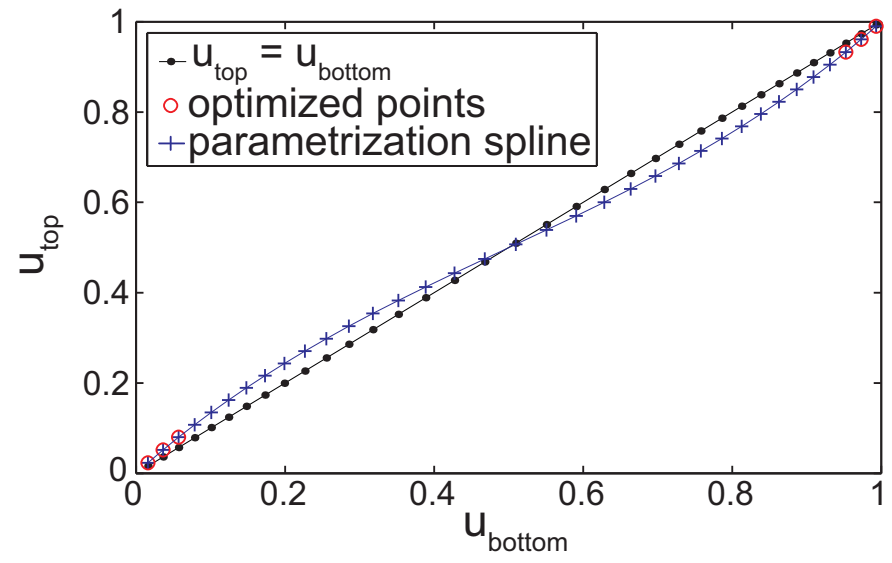

Figure 5: Parametrization spline

the procedure is repeated for the next point. Finally, the six optimized points are interpolated by a cubic B-Spline, see Fig. 5 . The smoothness of the cubic B-Spline ensures to have a smooth variation of the parametrization along the curve. Practically, the criterion based on the third derivatives is super sensitive to a tiny variation of the parametrization as it is shown in the next paragraph.

\subsection{Sensitivity analysis}

To demonstrate the high sensitivity of a tiny variation of the parametrization both on the criterion and the upper limit of the feedrate, the example detailed in section 4.1 is used. As shown on the left plot of Fig. 6, we are focusing only on the first orientation after the connection. So the first top point corresponding to $u_{t o p 1}$ has to be optimized. Indeed the bottom parametrization is fixed, so the bottom points are given (black dots in Fig. 6).

To study the sensitivity, the effect of the modification of the parameter $u_{t o p 1}$ on the criterion and on the upper limit of the feedrate is presented in Fig. 6. One can see that with a linear parametrization, $u_{\text {top } 1}=u_{\text {bottom } 1}$, the criterion has a really high value and the feedrate is limited to almost zero. An optimum can be found which allows to increase a lot the maximum reachable feedrate. However, a really tiny variation of the parameter induce a huge drop of the maximum reachable feedrate, see Fig. 6 right. Indeed, the third derivatives involved by the jerk limitations are really sensitive to a tiny variation of orientation. Because of this sensitivity, a special care should be paid to the Newton-Raphson optimization. But as the variation of the criterion is almost linear with only one minimum, this problem can be handled.

To conclude, this section presents a solution to solve the problem of orientation connection. Indeed, a special care should be paid to the continuity of the orientation even if the bottom and top curves are connected with a $G^{2}$ continuity, see Fig. 4. Using a parametrization spline linking the bottom and top parameters, this paper presents an efficient solution to the general problem of smooth orientation connection.

\section{Application}

The new algorithms for 5-axis corner rounding are applied on two examples. First, a 5-axis corner is rounded in a CAM context (with a large tolerance). It emphasizes the orientation connection problem studied in section 3. In this first example, comparisons with an industrial CNC are possible and show the benefits of the optimized orientation parametrization. Finally, a second example on a CNC context (with small contour tolerance) demonstrates that a jerk limited feedrate interpolation can be performed on the smoothed geometry.

\subsection{5-axis pocket with large corner rounding}

To demonstrate the need for the special attention paid to the derivatives of the tool orientation, an experiment has been carried out on a 5-axis high speed milling machine equipped with a Siemens 840D CNC. The kinematical limits of the drives can be found in [2]. A 5-axis corner is rounded using the proposed method with the bottom and top B-Spline curves. For this example, the contour and orientation tolerances are set to $3 \mathrm{~mm}$. This large tolerance emphasizes the problems linked to the linear parametrization $\left(u_{t o p}=u_{\text {bottom }}\right)$. Moreover, it makes possible to carry out experiments on an industrial $\mathrm{CNC}$ while controlling exactly the tool path with two B-Spline curves. Indeed, the Siemens 840D allows to programme a 5-axis tool path with a native cubic B-Spline format using the following code [3].

\section{BSPLINE SD $=3 \mathrm{~F}$ \\ $\mathrm{X}=\mathrm{Y}=\mathrm{Z}=\mathrm{XH}=\mathrm{YH}=\mathrm{ZH}=\mathrm{PL}=$}

The parameter "SD" gives the degree of the B-Spline curve, "F" gives the programmed feedrate along the curve, " $\mathrm{X}=\mathrm{Y}=\mathrm{Z}=$ " specifies the position of the bottom control points which defines the tool tip position. "XH= $\mathrm{YH}=\mathrm{ZH}=$ " specifies the position of the top control points which defines the tool orientation. Finally, "PL=" is the node vector. In this format, only one parameter is used for the nodal sequence so the bottom and top curves have the same parametrization $u_{\text {top }}=u_{\text {bottom }}$.

The 5-axis corner of an aluminium aeronautical pocket has been machined with a flat end mill $\phi 20 \mathrm{~mm}$ and a programmed feedrate of $F_{p r}=3000 \mathrm{~mm} / \mathrm{min}$, see Fig. 7. The results are presented on the left side of Fig. 8. On the top left plot, it is clear that the third derivatives of the orientation with respect to the path displacement are really high at the entrance and exit of the rounded portion. The middle left plot presents the upper limits of the feedrate, at the entrance and exit the measured feedrate on the 840D CNC is limited by the jerk constraints which lead to an almost zero feedrate at these points. Finally, the bottom left plot represents the measured feedrate on the surface swept by the tool axis. The localized feedrate slowdowns at the entry and exit of the transition are harmful for the surface finish because the cutting conditions are changing drastically along the flank of the workpiece. 

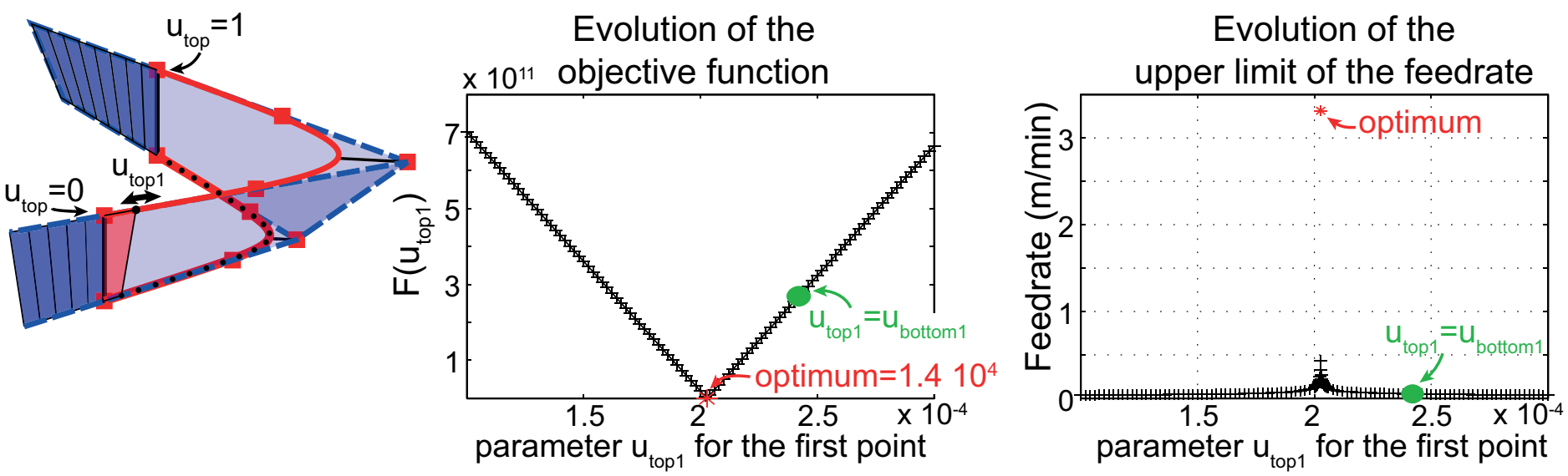

Figure 6: Effect of the position of the first point $u_{t o p 1}$ on the orientation connection

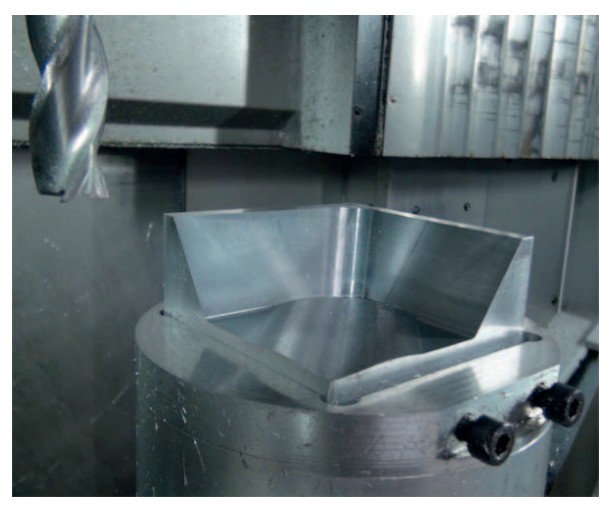

Figure 7: 5-axis machining of the large corner.

Using the optimized parametrization presented in this paper, it is possible to avoid these slowdowns due to a poor management of the evolution of the tool orientation. On the top right plot, one can see that the optimization procedure allows to avoid the large peaks on the derivatives of the orientation. In the middle right plot, a higher upper limit of the feedrate and thus a higher feedrate along the tool path are obtained. The VPOp feedrate presented here is computed within the context of an Open CNC. So the Velocity Profile Optimization software (VPOp) previously developed has been used to compute a feedrate which respects all the kinematical constraints of the 5 drives. It has been demonstrated in [2] that this feedrate interpolation algorithm is efficient and that the feedrate obtained is very similar to the feedrate measured on an industrial CNC. Finally, the bottom right plot shows that the programmed feedrate can be maintained at the entry and exit of the transition. Of course, in the middle of the corner there is no choice but to reduce the feedrate because the machine axes are not dynamic enough to handle the change of direction required by the tool path.

The optimized parametrization can reduce the machining time by half, see Fig. 9. So the productivity is increased as well as the surface finish as the feed marks are avoided. (a) $u_{\text {top }}=u_{\text {bottom }}$
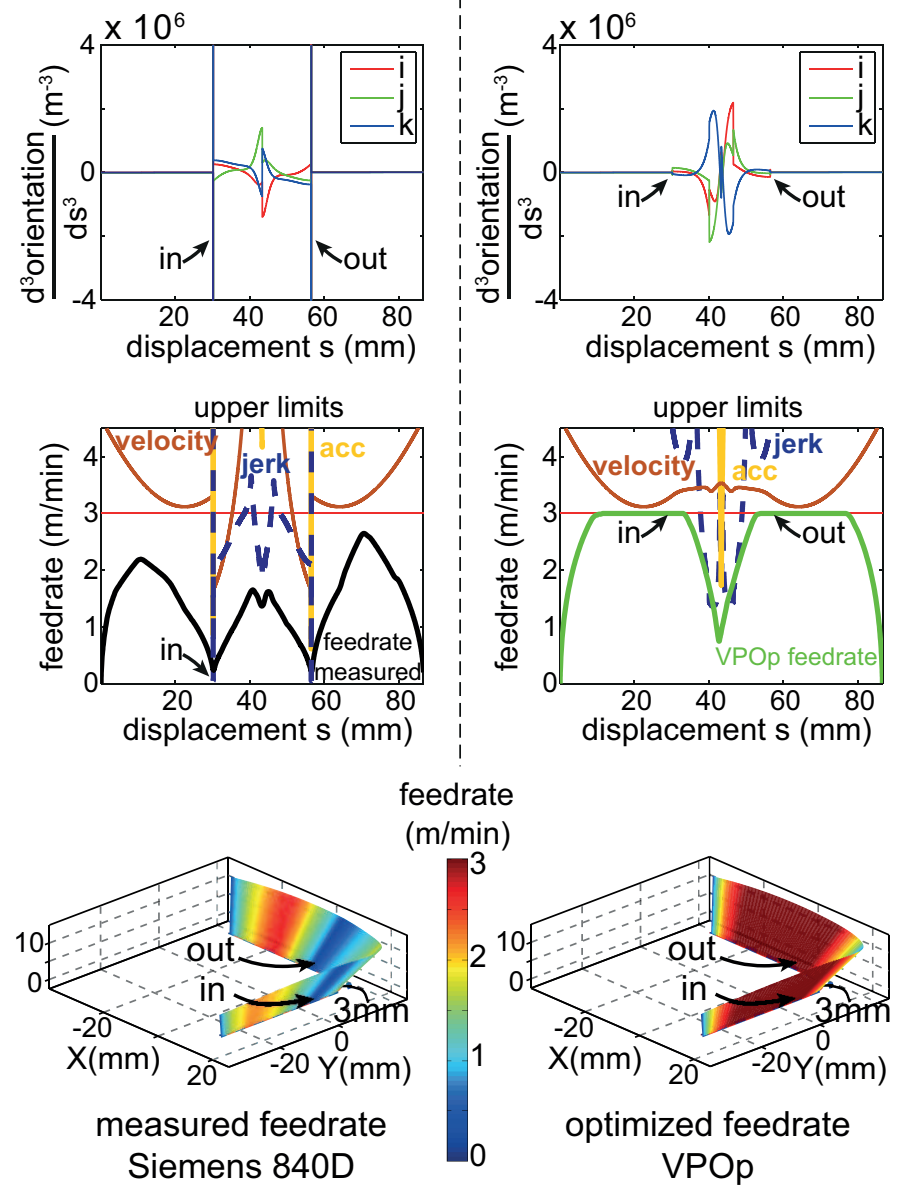

Figure 8: Presentation of the results for the 5-axis aeronautical pocket. Left: $u_{\text {top }}=u_{\text {bottom }}$ parametrization, right: optimized parametrization. 


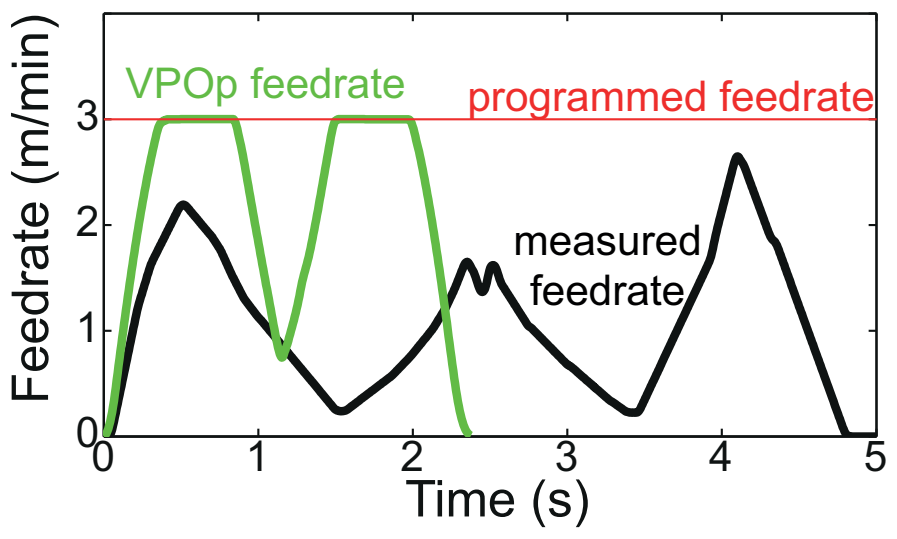

Figure 9: Comparison on the machining time for the aeronautical pocket.

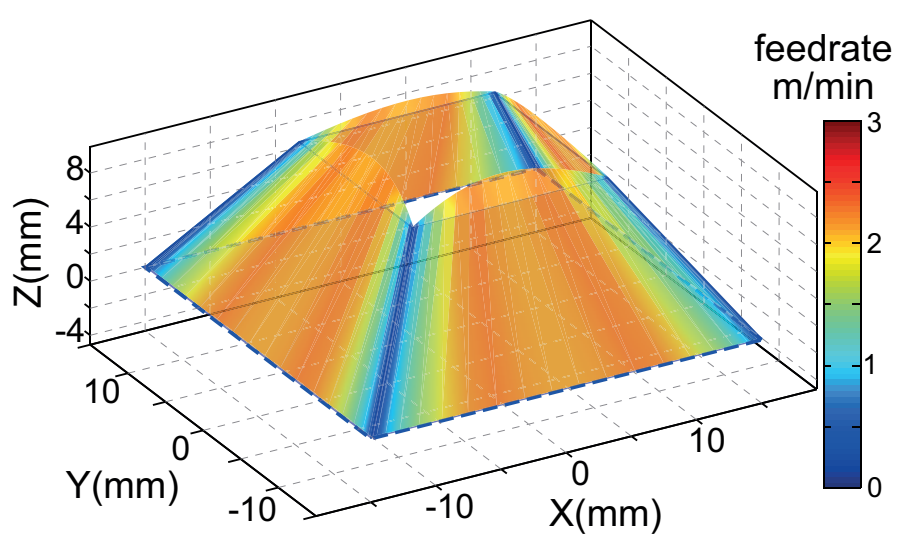

Figure 10: Pyramide surface with VPOp coloured feedrate.

\subsection{Pyramide with small corner rounding}

In this example, a pyramidal shape is machined in flank milling, see Fig. 10. Thus the tool path is described only by four segments. The contour tolerance is $0.02 \mathrm{~mm}$ which is a typical value used in the CNCs. The orientation tolerance is also $0.02 \mathrm{~mm}$. Unfortunately, with this kind of tolerance no comparison can be made with the Siemens CNC. Indeed, defining a tiny native cubic B-Spline is not appropriate here as the industrial CNCs are not designed to handle such a small geometry due to the interpolation cycle time for example.

Similarly to the previous example, the linear and optimized parametrization are compared in Fig. 11. A zoom is made around one corner as the others are identical. At this scale, the only limiting parameter is the axis jerk. One can see that the linear parametrization creates problems at the entrance and exit of the discontinuity both for the orientation derivatives and for the upper limit of the feedrate. Using the parametrization spline, it is possible to smooth the orientation connection and to remove these peaks.

Finally, once the geometry is optimized, it is possible to carry out the feedrate interpolation using VPOp as described in [2]. With a small contour tolerance, the feedrate interpolation is more difficult and without the optimized parametrization the
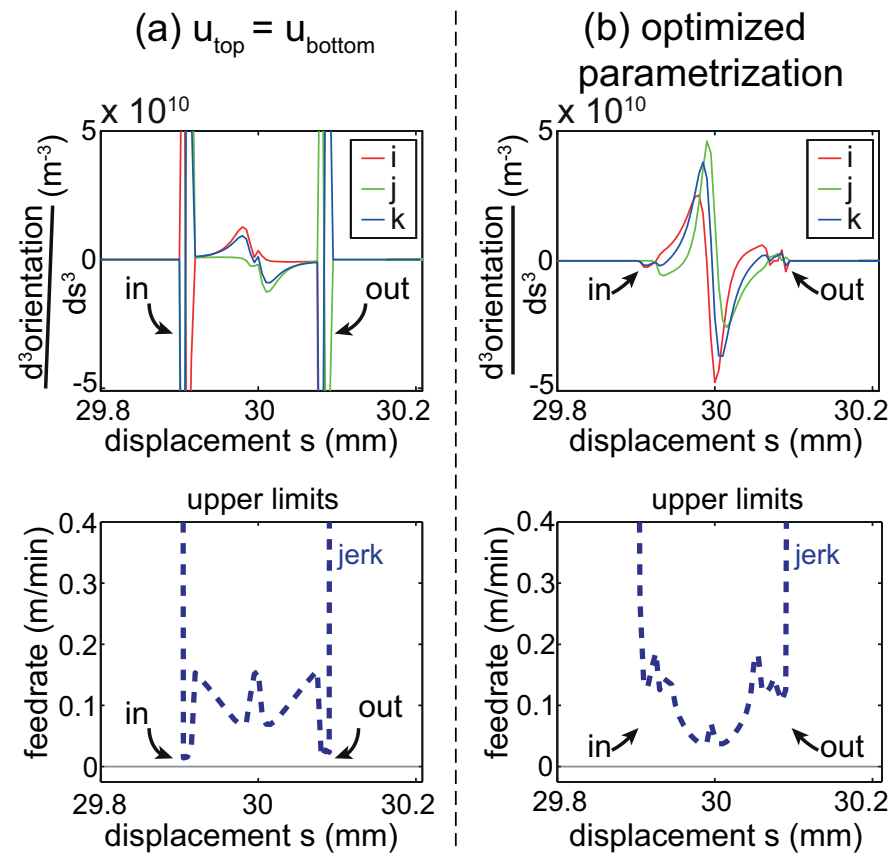

Figure 11: Presentation of the results for the pyramide. Left: $u_{t o p}=u_{\text {bottom }}$ parametrization, right: optimized parametrization.

jerk limitation is difficult to meet by the feedrate interpolation.

This example demonstrates that the proposed 5-axis corner rounding algorithms can be integrated in an industrial or Open $\mathrm{CNC}$ for a precise control of the workpiece geometry in the discontinuities.

\section{Conclusion}

To conclude, the most usual format used to programme 5axis tool path is the linear format (G1). Thus the discontinuities created at each transition between segments have to be rounded in order to perform the jerk limited feedrate interpolation.

After thorough study of the literature, the different tool path smoothing techniques are presented: local smoothing techniques are focused on 3-axis tool path and 5-axis tool path are only smoothed globally. Thus, in a first part, a 5-axis local corner rounding model is proposed. This model is based on two B-Spline curves to describe the tool path in the corner. By using this description, the contour and orientation tolerances can be controlled precisely. In a second part of the paper, the orientation connection between the linear portion and the corner is studied. Indeed, it is shown that the connection of two 5axis portions is critical and can lead to zero feedrate. So to solve the orientation connection problem, an optimized orientation parametrization is obtained with a parametrization spline. But, as it is shown in the first application, industrial CNCs lack the possibility to use a non-linear parametrization between the bottom and top curves. Furthermore, the examples also demonstrate that the proposed solution is multi-scale.

Finally, this paper brings two main contributions. First, a 5-axis 
corner rounding model is proposed whereas in the literature this problem was solved only in 3-axis. Second, the general problem of orientation connection of two 5-axis B-Spline tool paths is solved using the parametrization spline.

Further work could emphasize the need for an Open CNC able to control completely and precisely the geometry of the tool path in 5-axis. Thus the limitations brought by the CNC description formats could be overcome.

\section{Appendix A. Details on the 3-axis contour error}

The basis functions $B_{i j}$ can be defined using the Cox-de Boor recursion formula, Eq. A.1.

$$
\begin{aligned}
& B_{i 0}(u)=\left\{\begin{array}{l}
1, \text { if } u \in\left[u_{i}, u_{i+1}[\right. \\
0, \text { otherwise }
\end{array}\right. \\
& B_{i j}(u)=\frac{u-u_{i}}{u_{i+j}-u_{i}} B_{i, j-1}(u)+\frac{u_{i+j+1}-u}{u_{i+j+1}-u_{i+1}} B_{i+1, j-1}(u)
\end{aligned}
$$

With the nodal sequence, degree and number of control points used in the model presented in section 2.2, the following equations are obtained.

$$
\begin{aligned}
& B_{03}(u)= \begin{cases}(1-2 u)^{3}, & \text { if } u \in[0,0.5[ \\
0, & \text { if } u \in[0.5,1]\end{cases} \\
& B_{13}(u)= \begin{cases}2 u\left[(1-2 u)^{2}+(1-u)(2-3 u)\right], & \text { if } u \in[0,0.5[ \\
2(1-u)^{3}, & \text { if } u \in[0.5,1]\end{cases} \\
& B_{23}(u)= \begin{cases}2 u^{2}(3-4 u), & \text { if } u \in[0,0.5[ \\
2(1-u)^{2}(4 u-1), & \text { if } u \in[0.5,1]\end{cases} \\
& B_{33}(u)= \begin{cases}2 u^{3}, & \text { if } u \in[0,0.5[ \\
2(1-u)\left[u(3 u-1)+(2 u-1)^{2}\right], & \text { if } u \in[0.5,1]\end{cases} \\
& B_{43}(u)= \begin{cases}0, & \text { if } u \in[0,0.5[ \\
(2 u-1)^{3}, & \text { if } u \in[0.5,1]\end{cases}
\end{aligned}
$$

$B_{03}(0.5)=0$ and $B_{43}(0.5)=0$ so Eq. 2 can be obtained and the position of the points $\mathbf{P}_{\mathbf{0}}$ and $\mathbf{P}_{\mathbf{4}}$ does not affect the contour error.

Without loss of generality, the point $\mathbf{P}_{\mathbf{2}}$ can be the origin of the local coordinate system. Finally, the contour error is defined as follows and Eq. 3 can be obtained. The units vectors $\mathbf{v}$ and $\mathbf{w}$ are in the direction of $\mathbf{P}_{\mathbf{2}} \mathbf{P}_{\mathbf{1}}$ and $\mathbf{P}_{\mathbf{2}} \mathbf{P}_{\mathbf{3}}$, respectively.

$$
\varepsilon=\left\|\frac{1}{4} L_{1}(\mathbf{v}+\mathbf{w})\right\|
$$

\section{Appendix B. Geometrical derivatives of the orientation}

Starting from the orientation vector defined in Eq. 5, the first second and third geometrical derivatives are obtained.

The notation ' stands for the derivative of a function with respect to its own parameter, Eq. B.1. The function $f$ is the optimized parametrization which is unknown, $g$ reprensents the link between the parameter of the bottom curve and the path displacement, see Eq. B.2.

$$
\mathbf{C}_{t o p}^{\prime}=\frac{d \mathbf{C}_{t o p}\left(u_{t o p}\right)}{d u_{t o p}}
$$

$$
u_{\text {top }}=f\left(u_{\text {bottom }}\right) \quad, \quad u_{\text {bottom }}=g(s)
$$

From Eq.B.5, it is clear that $f^{\prime \prime \prime}$ should be bounded because $\mathbf{C}_{t o p}^{\prime} \neq 0$. Thus, at least a cubic polynomial form is required for the parametrization curve. Then, practical tests have shown that none of the terms in the equation can be neglected compared to others. So the Newton-Raphson optimization procedure presented in section 3.3 is required to obtain a smooth orientation connection.

$$
\begin{aligned}
\frac{d \mathbf{O r i}}{d s}= & \frac{1}{\left\|\mathbf{C}_{t o p}-\mathbf{C}_{\text {bottom }}\right\|} \cdot\left(\mathbf{C}_{\text {top }}^{\prime} f^{\prime}-\mathbf{C}_{\text {bottom }}^{\prime}\right) g^{\prime} \\
\frac{d^{2} \mathbf{O r i}}{d s^{2}}= & \frac{1}{\left\|\mathbf{C}_{t o p}-\mathbf{C}_{b o t t o m}\right\|} \cdot \\
& {\left[\left(\mathbf{C}_{t o p}^{\prime \prime} f^{\prime 2}+\mathbf{C}_{t o p}^{\prime} f^{\prime \prime}-\mathbf{C}_{b o t t o m}^{\prime \prime}\right) \cdot g^{\prime 2}\right.} \\
& \left.+\left(\mathbf{C}_{t o p}^{\prime} f^{\prime}-\mathbf{C}_{b o t t o m}^{\prime}\right) \cdot g^{\prime \prime}\right]
\end{aligned}
$$

$$
\begin{aligned}
\frac{d^{3} \mathbf{O r i}}{d s^{3}}= & \frac{1}{\left\|\mathbf{C}_{t o p}-\mathbf{C}_{\text {bottom }}\right\|} . \\
& {\left[\left(\mathbf{C}_{t o p}^{\prime \prime \prime} f^{33}+3 \mathbf{C}_{t o p}^{\prime \prime} f^{\prime} f^{\prime \prime}+\mathbf{C}_{t o p}^{\prime} f^{\prime \prime \prime}-\mathbf{C}_{b o t t o m}^{\prime \prime \prime}\right) \cdot g^{\prime 3}\right.} \\
& +\left(\mathbf{C}_{t o p}^{\prime \prime} f^{\prime 2}+\mathbf{C}_{t o p}^{\prime} f^{\prime \prime}-\mathbf{C}_{b o t t o m}^{\prime \prime}\right) \cdot 3 g^{\prime} g^{\prime \prime} \\
& \left.+\left(\mathbf{C}_{t o p}^{\prime} f^{\prime}-\mathbf{C}_{b o t t o m}^{\prime}\right) \cdot g^{\prime \prime \prime}\right]
\end{aligned}
$$

\section{Acknowledgments}

This work is supported by the French Ministry of Research and the Digiteo foundation (2010-47D).

\section{References}

[1] G. Pardo-Castellote, R. H. Cannon, Proximate time-optimal algorithm for on-line path parameterization and modification, IEEE International Conference on Robotics and Automation 2 (2) (1996) 1539-1546.

[2] X. Beudaert, S. Lavernhe, C. Tournier, Feedrate interpolation with axis jerk constraints on 5-axis NURBS and G1 tool path, International Journal of Machine Tools and Manufacture 57 (0) (2012) 73-82.

[3] Siemens, Sinumerik - 5 axis machining, DocOrderNo. 6FC5095-0AB10 0BP1 (2009).

[4] T. Otsuki, H. Ogino, S. Ide, T. Chiba, Curve interpolation method, Japan, Fanuc LTD. Yamanashi (US Patent 6823234 B2) (2004).

[5] M. Bouard , V. Pateloup, P. Armand, Pocketing toolpath computation using an optimization method, Computer-Aided Design 43 (9) (2011) 1099 1109.

[6] M.-S. Tsai, H.-W. Nien, H.-T. Yau, Development of a real-time lookahead interpolation methodology with spline-fitting technique for highspeed machining, The International Journal of Advanced Manufacturing Technology 47 (5-8) (2010) 621638.

[7] K. Shoemake, Animating rotation with quaternion curves, SIGGRAPH Comput. Graph. 19 (3) (1985) 245-254.

[8] M.-C. Ho, Y.-R. Hwang, C.-H. Hu, Five-axis tool orientation smoothing using quaternion interpolation algorithm, International Journal of Machine Tools and Manufacture 43 (12) (2003) 1259-1267.

[9] R. V. Fleisig, A. D. Spence, A constant feed and reduced angular acceleration interpolation algorithm for multi-axis machining, Computer-Aided Design 33 (1) (2001) 1-15. 
[10] W. Ge , Z. Huang, G. Wang, Interpolating Solid Orientations with a C2Continuous B-Spline Quaternion Curve, Technologies for E-Learning and Digital Entertainment 4469 (2007) 606-615.

[11] J. M. Langeron, E. Duc, C. Lartigue, P. Bourdet, A new format for 5-axis tool path computation, using bspline curves, Computer-Aided Design 36 (12) (2004) 1219-1229.

[12] P.-Y. Pechard, C. Tournier, C. Lartigue, J.-P. Lugarini, Geometrical deviations versus smoothness in 5-axis high-speed flank milling, International Journal of Machine Tools and Manufacture 49 (6) (2009) 454-461.

[13] X. Beudaert, P.-Y. Pechard, C. Tournier, 5-axis tool path smoothing based on drive constraints, International Journal of Machine Tools and Manufacture 51 (12) (2011) 958-965.

[14] S. Lavernhe, C. Tournier, C. Lartigue, Optimization of 5-axis high-speed machining using a surface based approach, Computer-Aided Design 40 (10-11) (2008) 1015-1023.

[15] C. Castagnetti, E. Duc, P. Ray, The Domain of Admissible Orientation concept: A new method for five-axis tool path optimisation, ComputerAided Design 40 (9) (2008) 938-950.

[16] S. J. Yutkowitz, W. Chester, Apparatus and method for smooth cornering in a motion control system, United States, Siemens Energy \& Automation, Inc. Alpharetta, GA (US Patent 6922606) (2005).

[17] K. Erkorkmaz, C.-H. Yeung, Y. Altintas, Virtual CNC system. Part II. High speed contouring application, International Journal of Machine Tools and Manufacture 46 (10) (2006) 1124-1138.

[18] V. Pateloup, E. Duc, P. Ray, Bspline approximation of circle arc and straight line for pocket machining, Computer-Aided Design 42 (9) (2010) 817-827.

[19] H. Zhao, L. Zhu, H. Ding, A real-time look-ahead interpolation methodology with curvature-continuous B-spline transition scheme for CNC machining of short line segments, International Journal of Machine Tools and Manufacture (2012) ONLINE FIRST.

[20] C. Ernesto, R. Farouki, High-speed cornering by CNC machines under prescribed bounds on axis accelerations and toolpath contour error, The International Journal of Advanced Manufacturing Technology 58 (1) (2012) 327-338.

[21] Q.Z. Bi, Y. H. Wang, L. M. Zhu, H. Ding, A Practical ContinuousCurvature Bezier Transition Algorithm for High-Speed Machining of Linear Tool Path, Intelligent Robotics and Applications 7102 (2011) 465476. 